\title{
The Influence of Time-of-Flight (TOF) Mass Spectra Peak Resolution on Elemental Detection Limits for Atom Probe Instrumentation.
}

\author{
P.H. Clifton, ${ }^{*}$ A. Cerezo, ${ }^{* *}$ and G.N.F. Chapman* \\ * Oxford nanoScience Ltd, 4-6 Carters Lane, Kiln Farm, Milton Keynes, UK, MK11 3ER \\ **Department of Materials, Oxford University, Parks Road, Oxford, UK, OX13PH
}

The unique capabilities of the three dimensional atom probe technique (3DAP) are due to a combination of high spatial resolution single-ion detection allied with the ability to determine the chemical identity of atoms within an analysed volume [1]. For accurate chemical composition to be obtained it is vital that the mass spectra are of sufficient quality to allow peaks with mass differences as small as $0.33 \mathrm{amu}$ to be fully resolved. This necessitates mass resolution values in excess of $300(\mathrm{~m} / \Delta \mathrm{m})$ to be achieved and full-width at half-maximum (FWHM) values in excess of 500 are quoted for commercially available systems. However, this parameter does not adequately describe the ability of the 3DAP to resolve small peaks lying close to those of major constituents. This is a particular problem in engineering alloys, where important alloying additions often lie close to the main peak, for example that due to $\mathrm{Fe}, \mathrm{Al}$ or $\mathrm{Ni}$ according to the alloy type. Full-width at tenth maximum (FWTM) mass resolution is a better measure of the ability of an instrument to separate peaks in such cases. The introduction of energy compensation has greatly reduced the high-mass tailing observed with standard instruments and produced significant improvements in FWTM mass resolution [2].

A section of a mass spectrum is shown in Fig. 1 from the analysis of a stainless steel obtained using an energy-compensated Oxford nanoScience 3DAP instrument. Resolution values of 560 (FWHM) and 350 (FWTM) were measured from the main $\mathrm{Fe}^{2+}$ peak. Values could also be calculated for the full-width at one-hundredth and one-thousandth maximum (147 and 97 respectively). In this example the heights of the ${ }^{58} \mathrm{Ni}^{2+}$ and ${ }^{59} \mathrm{Co}^{2+}$ peaks have a ratio of approximately 20 , and can clearly be seen to be fully resolved.

In an atom probe without energy compensation, the tail of each peak is typically found to have an exponential decay [3]. Based on this, a model for 2 peaks in the mass spectrum was constructed and the resulting percentage of peak overlap calculated for different values of FWTM mass resolution. This showed that in the case of Ni and Co peaks a FWTM value of at least 130 was required in order to successfully resolve the two peaks. However, for smaller levels of the second peak higher FWTM resolutions would be needed to avoid significant overlap (Fig. 2). Based on the same peak model, the minimum values of FWTM resolution were calculated which would give no more than a $10 \%$ contribution to the second peak intensity from the dominant species, as shown in Fig. 3. FWTM resolutions of better than 230 are required in order to enable detection limits substantially above a peak ratio of 1000, strongly supporting the need for energy compensated atom probe instrumentation for the accurate determination of chemical compositions within complex material systems.

References

[1] M.K. Miller et al., 'Atom Probe Tomography', Plenum Press, New York, 2000

[2] A. Cerezo et al., Rev. Sci. Instrum. 69 (1998) 49-58.

[3] M.K. Miller and G.D.W. Smith, 'Atom Probe Microanalysis: Principles and Applications to Material Problems’ MRS, Pittsburgh, 1989 

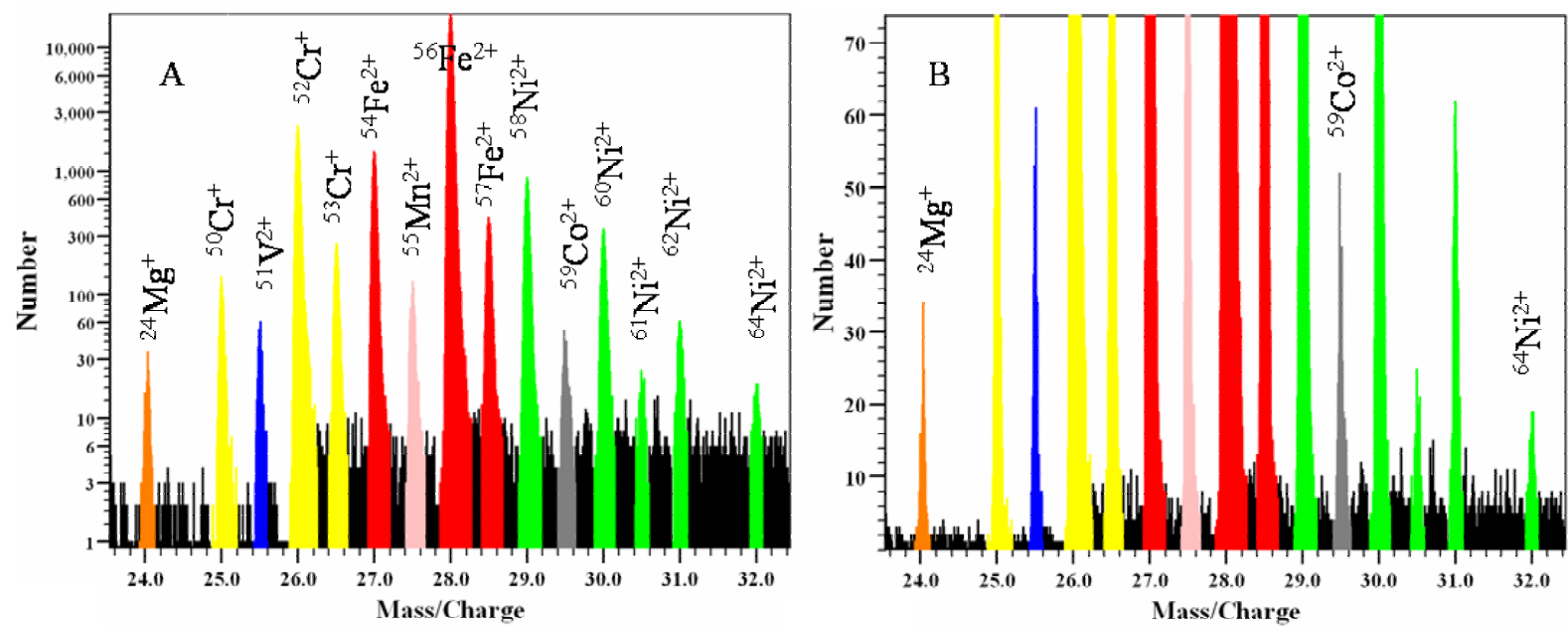

FIG. 1. 3DAP mass spectra obtained from a steel specimen displayed between 23 and 33 amu with A) a $\log$ scale used and B) a linear scale showing the base of the peaks. In this example the ${ }^{58} \mathrm{Ni}^{2+}$ peak and ${ }^{59} \mathrm{Co}^{2+}$ peaks are separated by $0.5 \mathrm{amu}$ and have a ratio of peak intensity of $20: 1$.
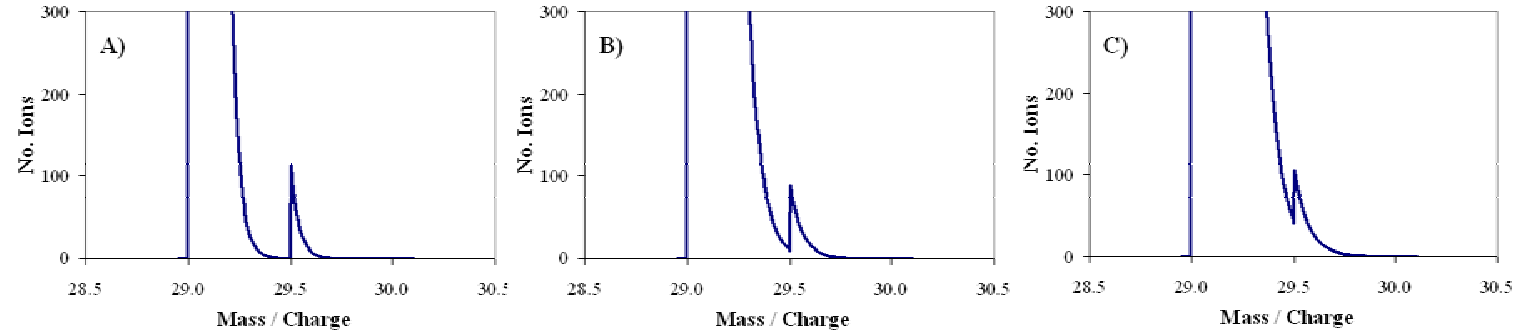

FIG. 2. Model data illustrating the effect of FWTM variation for a peak ratio of 1000 where A) FWTM is 350 and the peaks are fully resolved, B) FWTM is 230 and $10 \%$ of the second peak intensity is due to overlap and C) where 50\% overlap has occurred for a FWTM value of 190 .

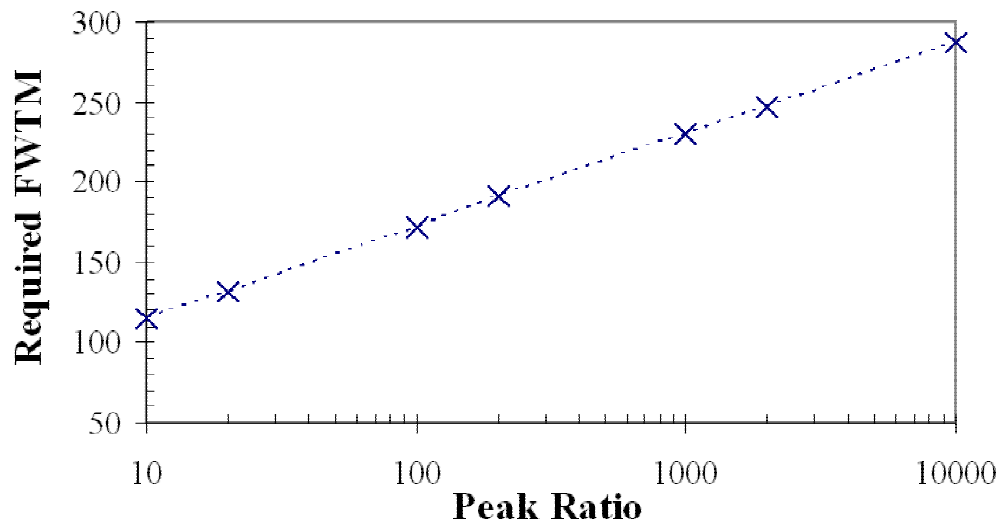

FIG. 3. Plot of the FWTM required in order for the two species to be resolved as a function of the peak ratio. 